DOI: 10.4274/jarem.galenos.2020.3354

J Acad Res Med 2020;10(3):294-7

\title{
T-Tube Breakage During Removal: Management by Endoscopic Retrograde Cholangiopancreatography
}

\author{
(1) Mehlika Bilgi Kırmacı1', (1) Tamer Akay22, (1) Esra Özgül3, (1) Sezgin Yılmaz1 \\ ${ }^{1}$ Afyonkarahisar University of Health Sciences Faculty of Medicine, Department of General Surgery, Afyonkarahisar, Turkey \\ ${ }^{2}$ Bandırma State Hospital, Clinic of General Surgery, Balıkesir, Turkey \\ ${ }^{3}$ Afyonkarahisar University of Health Sciences Faculty of Medicine, Department of Radiology, Afyonkarahisar, Turkey
}

Cite this article as: Bilgi Kırmacı M, Akay T, Özgül E, Yılmaz S. T-Tube Breakage During Removal: Management by Endoscopic Retrograde Cholangiopancreatography. J Acad Res Med 2020;10(3):294-7

\begin{abstract}
T-tube drainage is a widely used procedure among surgeons for various biliary conditions. However, various complications may occur, especially due to the breaking-off of the T-tube from the area where it enters the common bile duct during retraction. These complications often require subsequent surgical procedures to remove the T-tube fragment from the common bile duct lumen. In selected cases, the T-tube fragment can be extracted from the common bile duct by endoscopic retrograde cholangiopancreatography (ERCP). ERCP has a life-saving role that eliminates reoperation for such a complication. Herein, we present a 42-year-old female patient who was referred to our hospital, which is as an advanced center, due to breakage during T-tube withdrawal. The patient had previously undergone open cholecystectomy and T-tube drainage. When removing the T-tube, a breakage occurred at 3-4 cm from the T-tube leg, which was left in the common bile duct. In the ERCP, the leg of the T-tube was removed from the common bile duct lumen by basket compression following endoscopic sphincterotomy and a temporary plastic stent was placed. One month after the procedure, the stent was removed with the help of the ERCP. This case is presented as a rare indication of ERCP.
\end{abstract}

Keywords: Cholangiopancreatography, endoscopic retrograde, drainage, T-tube, complications

\section{INTRODUCTION}

Endoscopic retrograde cholangiopancreatography (ERCP) is widely used in the diagnosis and treatment of biliary tract and pancreatic diseases. Performed for diagnostic and therapeutic purposes, this procedure has a life-saving role in most cases. Although it is generally used for several biliary and pancreatic diseases, certain rare cases also require its use for therapeutic intentions (1). T-tube is still a matter of debate, even though Huang et al. showed in the largest meta-analysis that the insertion of a T-tube may be useful. Insertion of a T-tube reduces the incidence of biliary strictures. However, it also has adverse events, predominately leakage, peritonitis, and cholangitis. The T-tube is typically removed through the percutaneous route. However, this could be impossible, probably due to fibrotic and scarring processes. Those cases are classically treated by surgery. Endoscopic removal by ERCP is a minimally invasive approach that avoids a laparotomy (2-4). In this paper, we present a 42-year-old woman who was admitted to our ERCP unit for T-tube breakage during removal.

\section{CASE PRESENTATION}

The 42-year-old woman had undergone open cholecystectomy and T-tube drainage for biliary lithiasis at another hospital

ORCID IDs of the authors: M.B.K. 0000-0002-8034-1459; T.A. 0000-0001-8137-3658; E.Ö. 0000-0002-6005-134X; S.Y. 0000-0003-0213-3758. 
40 days ago. While the surgeon was taking out the T-tube, it was broken at the junction of the head and limp. The cup part of the tube remained in the common bile duct lumen, and the patient was referred to our ERCP unit for the removal of the leg of the T-tube. The patient had a mild abdominal pain and tenderness. The biochemical parameters were in the normal range. Abdominal magnetic resonance and magnetic resonance cholangiopancreatography images revealed that the tube fragment was located in the common bile duct (Figure 1, 2).

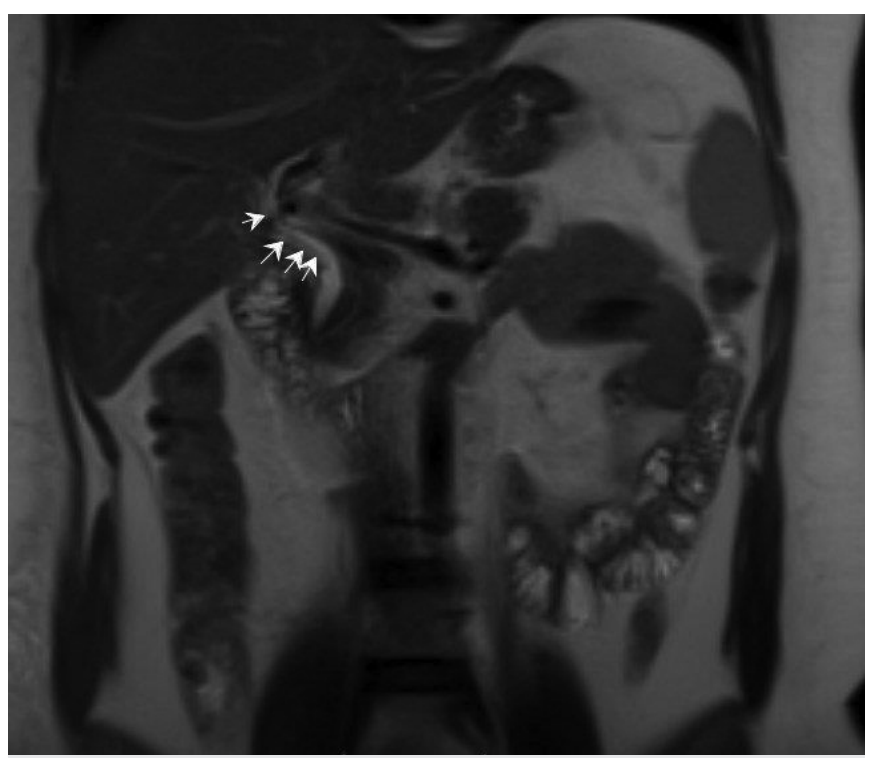

Figure 1. Coronal thin-sliced abdominal MR shows a linear filling defect extending from the distal right intrahepatic bile duct to the proximal common bile duct

MR: magnetic resonance

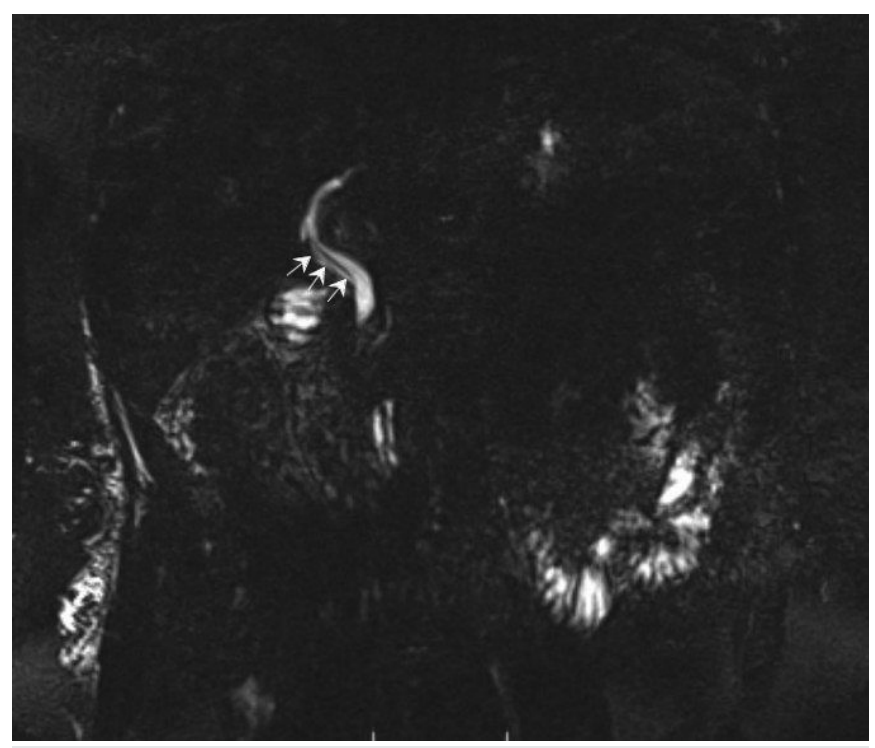

Figure 2. MRCP sequence shows that the common bile duct is $8 \mathrm{~mm}$ in diameter and that the breakage of the leg of the T-tube is in common bile duct

MRCP: magnetic resonance cholangiopancreatography
Informed consent was obtained from the patient after providing detailed information about the surgery.

ERCP was performed and the common bile duct was cannulated using a sphincterotome and guide-wire. There was a linear-tubular filling defect in the common bile duct lumen, compatible with the residual fragment of the T-tube (Figure 3). A $20 \mathrm{~mm}$ sphincterotomy was performed using a rounded sphincterotome and guide-wire. The common bile duct was swept using a basket. At this time, the residual fragment was extracted (Figure 4, 5).

A $10 \mathrm{Fr} 12 \mathrm{~cm}$ plastic stent was placed into the common bile duct lumen. No pathologic finding was seen on control ERCP cholangiogram. A transient biliary stent was placed and the patient was discharged two days after the procedure.

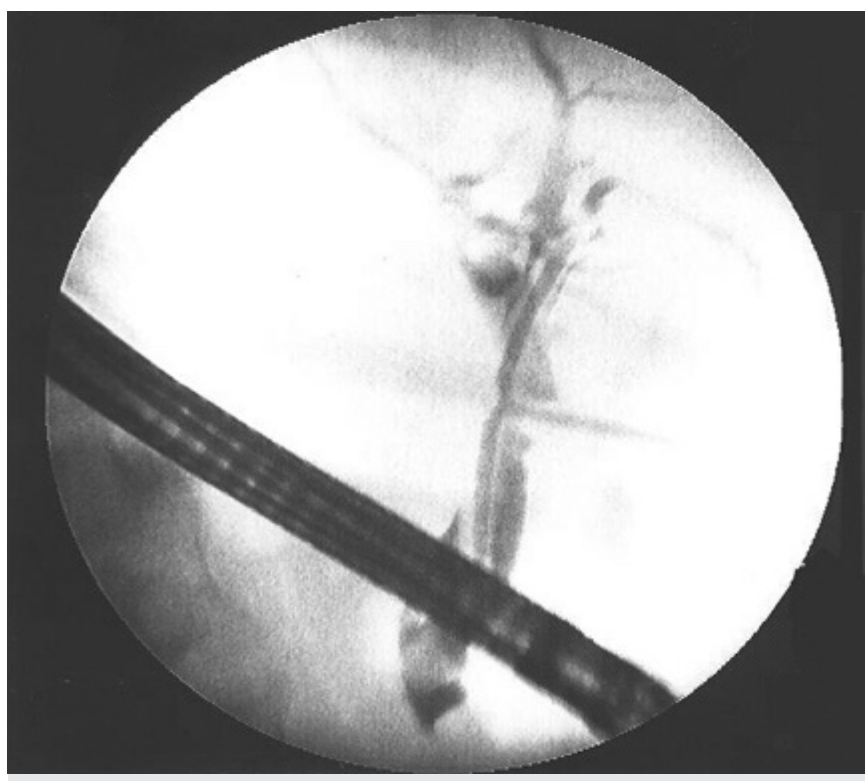

Figure 3. ERCP shows a linear-tubular filling defect in the common bile duct lumen

ERCP: endoscopic retrograde cholangiopancreatography

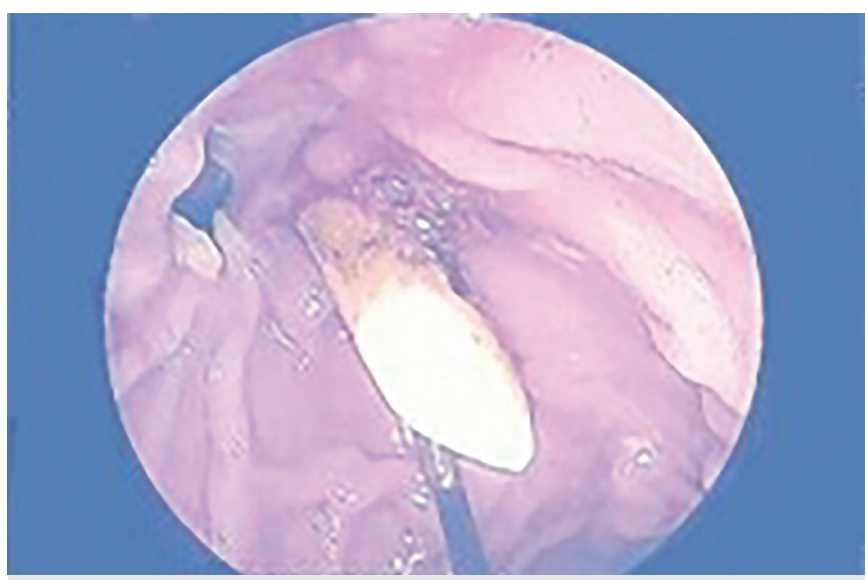

Figure 4. Endoscopic basket extraction of the residue T-tube fragment by ERCP

ERCP: endoscopic retrograde cholangiopancreatography 


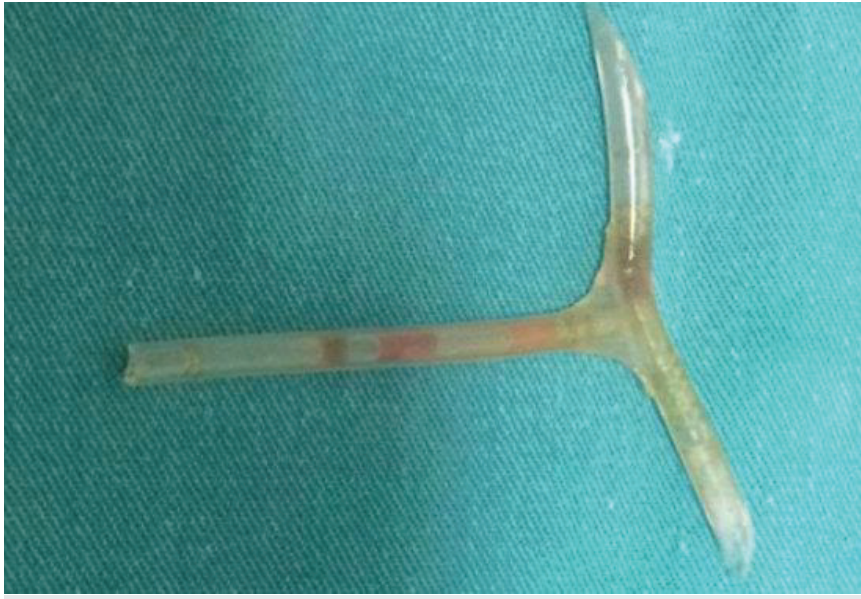

Figure 5. Retained T-tube fragment.

ERCP was successfully performed to remove the leg of the T-tube and the patient was discharged without any problem.

\section{DISCUSSION}

Common bile duct stones constitute an extended period in which ERCP is usually applied as the first-line therapy. However, ERCP cannot remove larger stones from the sphincterotomy area. Therefore, open surgery is required for such cases (5). There are several methods that can be applied during open surgery following the extraction of stones from bile ducts, such as biliaryenteric anastomosis, primary suturing of the bile duct wall and T-tube drainage.

Patients under 65 years of age who had dilated bile ducts below 15 $\mathrm{mm}$ in diameter are candidates for T-tube placement. The T-tube can be removed following a period of 4 days- 2 weeks depending on the preference (6).

Although T-tube drainage is a commonly used procedure, it has several complications resulting in biloma, bile ascites, cholangitis, and peritonitis. These complications may occur due to bile leak following T-tube removal. In addition, there are other rare complications that occur during the accidental breakage from the insertion site into the bile duct during T-tube limb removal. This may be caused by severe adhesions of the tube as well as suturing of the T-tube to the duct channel (7). Sharma and Farah (8) reported nine cases of T-tube fragment retained in the common bile duct. They were removed immediately in four cases (two cases with ERCP, one case with percutaneous radiological approach, one case with surgery), since the T-tube was known to have been fractured during removal. However, the other five cases remained asymptomatic for 2-36 years until cholangitis appeared (8).

For cases where the T-tube fragment remains asymptomatic for a certain period, patients may have unexplained abdominal pain, jaundice, cholestasis, and related symptoms. T-tube fragments retained in the common bile duct lumen should be taken out immediately the diagnosis is made. Radiological, surgical, and endoscopic procedures are alternatives to removing such fragments. However, ERCP should be the initial procedure in the management, if possible. Surgical procedures should be scheduled only if radiological or endoscopic interventions fail (9).

Although primary closure of the common bile duct is safer and more effective than T-tube drainage, there is still a wide use of T-tube after choledochotomy among surgeons. Choleperitonitis is one of the most severe complications resulting from leakage of bile from the choledochotomy site due to insufficient fistula formation after removal (10). In our opinion, these complications are more frequent than mentioned among surgeons, since most of them are not reported.

Other rare complications caused by T-tube breakage during removal occur mainly due to the improper suturing of the duct to the channel or inflammatory reactions to the drainage material. Thus, the tube fragment remains in the common bile duct lumen. In this case, the presentation is quite variable depending on the clinical status of the patient and texture of the drain. Although patients may remain asymptomatic for years, bile duct stones, cholangitis, and even common bile duct injury can be seen early or late after the breakage. Measures that could be taken to reduce the risk of T-tube breakage during removal include careful T-tube preparation, prevention of partial separation, use of latex drains, preventing T-tubes from being accidentally erected in the channel of the wall, and preventing prolonged placement (11).

Retention of T-tube fragments upon removal requires immediate surgery to avoid potential serious complications. ERCP should be the initial step to remove such fragments in centers where it is available. Otherwise, surgical approach will be mandatory, but re-exploration of the biliary tract increases surgical complications, especially bile fistula and stricture formation (12).

\section{CONCLUSION}

Broken T-tube fragments should be removed immediately to avoid potentially fatal complications due to the breaking of the T-tube fragments. The first step to avoid reoperation is to intervene endoscopically.

Informed Consent: Informed consent was obtained from the patient after providing detailed information about the surgery.

Peer-review: Externally peer-reviewed.

Author Contributions: Surgical and Medical Practices - M.B.K., T.A.; Concept - S.Y., E.Ö.; Design - M.B.K., S.Y.; Data Collection or Processing E.Ö.; Analysis or Interpretation - S.Y., E.Ö.; Literature Search - T.A., M.B.K.; Writing - T.A.

Conflict of Interest: The authors have no conflict of interest to declare.

Financial Disclosure: The authors declared that this study has received no financial support.

\section{REFERENCES}

1. Daldoul S, Mabrouk A, Messoudi I, Ben Dhaou A, Sayari S, Baccari A, et al. Multiple bile duct stones: Comparison of two surgical techniques done at laparotomy. Tunis Med 2016; 94: 643. 
8. Sharma T, Farah KF. A 36-year retained T-tube fragment presenting with cholangitis. Gastroenterology Res 2009; 2: 126-7.

9. Daldoul S, Moussi A, Zaouche A. T-tube drainage of the common bile duct choleperitoneum: etiology and management. J Visc Surg 2012; 149: e172-8.

10. Nikolić M, Karthikesalingam A, Nachimuthu S, Tang TY, Harris AM. Biliary peritonitis caused by a leaking T-tube fistula disconnected at the point of contact with the anterior abdominal wall: a case report. J Med Case Rep 2008; 16: 302.

11. Haq A, Morris J, Goddard C, Mahmud S, Nassar AH. Delayed cholangitis resulting from a retained $\mathrm{T}$-tube fragment encased within a stone: a rare complication. Surg Endosc 2002; 16: 714.

12. Wang $L$, Dong $P$, Zhang $Y$, Liu $X$, Tian B. latrogenic bile duct Injury with a retained T-tube in common bile duct for 10 years: A case report. Medicine (Baltimore) 2019; 98: e15127. 